Management international

International Management

Gestiòn Internacional

\title{
La délégation managériale. Levier de compétences et de développement stratégique
}

\section{Ewan Oiry}

Volume 23, numéro 4, 2019

URI : https://id.erudit.org/iderudit/1066080ar

DOI : https://doi.org/10.7202/1066080ar

Aller au sommaire du numéro

Éditeur(s)

HEC Montréal

Université Paris Dauphine

ISSN

1206-1697 (imprimé)

1918-9222 (numérique)

Découvrir la revue

Citer ce compte rendu

Oiry, E. (2019). Compte rendu de [La délégation managériale. Levier de compétences et de développement stratégique]. Management international/ International Management / Gestiòn Internacional, 23(4), 191-192.

https://doi.org/10.7202/1066080ar d'utilisation que vous pouvez consulter en ligne.

https://apropos.erudit.org/fr/usagers/politique-dutilisation/ 


\title{
La délégation managériale. Levier de compétences et de développement stratégique
}

\author{
AUTEUR: DIDIER RETOUR
}

Editions: Vuibert, 2015

EWAN OIRY

ESG-UQAM

Le 'ouvrage «La délégation managériale. Levier de compétences et de développement stratégique» est particulièrement important et original.

Tout d'abord, le thème de la délégation est particulièrement important sur le plan managérial. Dans tous les types d'entreprise, la question de la délégation se pose. Dans les très grandes entreprises privées comme publiques, dans les PME comme dans les plus petites organisations de l'économie sociale, la question se pose de ce qui doit être délégué, de ce qui peut être délégué et, enfin, de comment cette délégation peut s'organiser concrètement.

Ensuite, cet ouvrage est original parce que le thème de la délégation managériale est peu travaillé par la littérature scientifique. Il existe quelques ouvrages plutôt prescriptifs qui proposent des «recettes » pour une délégation managériale réussie mais cet ouvrage propose d'utiliser les résultats de la recherche en management et en Gestion des Ressources Humaines pour conceptualiser la délégation et en tirer des préconisations à destination des managers. A titre personnel, chaque manager et, même chaque employé, s'est posé la question de la délégation : que puis-je déléguer? A qui? Dans quelles conditions? Comment faire pour que déléguer ne soit pas «se débarrasser»? Comment puis-je être certain que ce que j'ai délégué sera bien fait? Aussi bien (ou même mieux?) que si je le faisais moi-même?

Enfin, cet ouvrage renouvelle profondément la réflexion sur la délégation managériale en proposant de ne pas la considérer de manière isolée, comme un rôle que chaque manager doit apprendre mais de la considérer comme inscrite dans l'écosystème de l'organisation. Comme l'indique le sous-titre de l'ouvrage, la délégation managériale articule le niveau individuel du développement des compétences avec le niveau des compétences et des objectifs stratégiques de l'organisation.

Cet ouvrage conceptualise donc la délégation managériale comme un processus qui met en dynamique les niveaux individuels du manager et de son équipe avec le niveau stratégique de l'organisation qui, grâce à ses compétences, parvient à se positionner sur son marché et proposer des produits et services innovants à ses clients.

Regardons maintenant plus en détail comment cet ouvrage est organisé pour relever ce défi de mieux conceptualiser la délégation managériale pour proposer des moyens d'action concrets pour les managers dans les organisations.

Le premier chapitre de cet ouvrage démontre l'importance de la délégation managériale en la positionnant immédiatement dans son contexte organisationnel et stratégique. Didier Retour montre ici que la délégation managériale est une dimension indispensable dans l'action du manager lorsque celui-ci se trouve dans une organisation flexible, où les individus comme les équipes sont de plus en plus autonomes, où le nombre de niveaux hiérarchiques est réduit, où le niveau de formation et les compétences des employés sont en croissance régulière et, où, finalement, le management participatif est de plus en plus présent. Ce premier chapitre positionne donc clairement la délégation managériale comme un des ingrédients centraux de l'avantage concurrentiel des entreprises qui adoptent des modèles organisationnels innovants.

Le second chapitre s'attache à définir la délégation managériale. Ce chapitre est le plus long de l'ouvrage car la délégation managériale prend des formes multiples. Ces formes doivent être analysées avec attention car chacune à sa pertinence, ses avantages et ses limites. C'est toute la palette des délégations managériales qu'il convient de saisir pour utiliser de manière optimale cet outil dans les organisations innovantes.

Initialement, la définition générale de la délégation peut sembler assez simple. Didier Retour la définit (p. 22) de la manière suivante : «En vue d'assurer le développement des compétences des salariés et de l'entreprise, acte de management consistant à confier temporairement à une personne (le délégué) tout ou partie d'une activité normalement assurée, dans le cadre de son périmètre de poste, par le délégant qui en garde la responsabilité».

Bien que relativement simple, cette définition contient plusieurs caractéristiques importantes. Tout d'abord, la délégation managériale est considérée comme un levier de développement des individus (les employés, le délégué comme le délégant) mais aussi de l'organisation. Ensuite, la délégation est temporaire. Nous verrons en fait que, de manière très intéressante, Didier Retour propose de la penser comme un processus. Si toute délégation doit initialement être clairement temporaire, en revanche, il est possible d'en penser un mode d'accompagnement qui, grâce au développement progressif des compétences du délégué et au soutien sans faille du délégant, rend la délégation permanente (on aboutit alors à un enrichissement de fonction). Enfin, il est crucial de noter que dans la délégation, ce sont les "activités " qui sont déléguées. Les responsabilités restent aux mains du délégant. La délégation managériale ne consiste pas à se débarrasser d'activités que le délégant ne souhaite pas faire : il reste responsable de ce qui a été réalisé par le délégué. 
Ce chapitre de définition est long parce qu'au-delà de la délégation managériale «simple» où un manager confie à un de ses subordonnés une activité qui était jusqu'alors inscrite dans son périmètre de poste, il existe de très nombreuses formes différentes de la délégation. Chacune a ses attraits et ses difficultés. Par exemple, la délégation managériale peut concerner des activités de diagnostic, de recherche de solution, de mise en œuvre ou de contrôle. Elle peut être étroite ou large (on retrouve ici la notion de processus qui permettra de passer de la délégation qui est temporaire à l'enrichissement de fonction rendu possible par le développement des compétences du délégué). La délégation peut être horizontale (vers un collègue de niveau hiérarchique équivalent). On peut également envisager la sous-délégation (le délégué délègue lui-même l'activité) et même la délégation «ascendante» (bottom-up) : c'est un subordonné qui délègue une activité à son superviseur!

P.44, Didier Retour propose une synthèse des caractéristiques de la délégation. Nous ne pouvons toutes les reprendre ici mais, juste en en citant quelques-unes, nous constaterons la complexité et l'importance stratégique de cet acte managérial. La délégation managériale s'effectue de personne à personne (relation interpersonnelle forte et de qualité), elle porte sur des activités pas sur des responsabilités, elle doit être négociée et acceptée, elle reconnaît un droit à l'erreur, elle favorise le développement de nouvelles compétences, etc.

Le troisième chapitre décrit les avantages et les risques de ces différentes formes de délégations. En complète congruence avec le propos global de l'ouvrage, Didier Retour identifie ces avantages et ces risques à un triple niveau : celui du délégué, celui du déléguant (et donc en fait celui de leur relation) et celui de l'organisation. A nouveau ici, la délégation managériale est positionnée comme un levier de développement des individus et de la stratégie de l'organisation. La confiance, la reconnaissance, le développement personnel et professionnel, l'intérêt accru pour le travail, toutes ces potentialités, pour le délégué, de la délégation managériale sont évoquées. Pour le déléguant comme pour l'organisation, pour circonscrire les risques, l'enjeu est de réussir à proposer au délégué un processus de soutien et d'accompagnement qui lui permette de réaliser les activités qui lui ont été confiées et de développer les compétences nécessaires pour les réaliser de manière durable.

L'ensemble de l'ouvrage est enrichi de très nombreux encadrés présentant des témoignages d'entreprises et de managers. Régulièrement, Didier Retour pose même des questions au lecteur pour l'engager dans une posture réflexive d'analyse de ses propres pratiques de délégation et des besoins de son organisation sur ce thème. Le quatrième et dernier chapitre est consacré au cas d'une entreprise, celle qui a déclenché chez Didier Retour l'envie et la nécessité de proposer une conceptualisation de la délégation managériale. A partir d'entretiens avec les employés, en fonction des managers et des filiales, il y identifie quatre types de délégation : la délégation-fonctionnement (elle est nécessaire pour la production), la délégation utilitariste (elle accroit l'efficacité), la délégation personnalisée (elle permet d'accroître les compétences du délégué) et la délégation-développement (elle permet le développement des individus, des équipes et de l'organisation). Les outils de positionnement construits à partir de cette typologie permettent de développer des tableaux de bord qui permettent de développer une réflexion stratégique sur la délégation managériale. La présentation détaillée de ce cas d'entreprise permet donc, à la fin de cet ouvrage, de voir comment sa thèse centrale (la délégation managériale est un levier de développement des individus et des organisations), peut s'incarner dans des pratiques managériales et la stratégie d'une organisation.

Cet ouvrage est donc à la fois original, intéressant et une ressource pour la réflexion comme pour l'action. La FNEGE ne s'y est d'ailleurs pas trompée : elle l'a inscrit dans sa liste des meilleurs ouvrages en management.

Nous incitons tous les lecteurs (managers, étudiants comme chercheurs) à le lire avec attention! 